\title{
Pathologic Changes in a Rabbit Model of Reye's Syndrome
}

\author{
DORIS A. TRAUNER ${ }^{(18)}$ \\ Departments of Neurology and Pediatrics, University of California School of Medicine, La Jolla, California, USA
}

\begin{abstract}
Summary
Continuous infusion of the short-chain fatty acid sodium octanoate into rabbits produces pathologic changes after $\mathbf{4 h}$. Principal abnormalities include microvesicular fatty accumulation in liver, and to a lesser extent in kidney, heart, and lungs. Ultrastructural changes include swelling and pleiomorphism of mitochondria in liver, and less consistently swelling of astrocytic foot processes in brain, suggesting early cerebral edema. These changes are similar to the pathologic abnormalities observed in patients with Reye's syndrome.
\end{abstract}

\section{Speculation}

Infusion of the short-chain fatty acid anion, octanoate, into rabbits reproduces the major clinical, biochemical, and pathologic changes observed in Reye's syndrome, and thus serves as a suitable experimental model for the human disease.

Reye's syndrome (10) is an acute metabolic encephalopathy, which occurs primarily in children although recently adult cases have been recognized (16). Clinical features include an antecedent viral illness followed by vomiting and progressing rapidly to delirium, coma, hyperventilation, and seizures. The disease has a significant mortality rate despite advances in supportive care. Biochemical abnormalities include hyperammonemia, elevated liver enzymes, prolonged prothrombin time, hypoglycemia, hyperamino-acidemia and short-chain fatty acidemia (6). Pathologic changes consist primarily of microvesicular fatty accumulation in the liver, liver mitochondrial swelling, and cerebral edema $(6,9)$.

The underlying cause of Reye's syndrome is unknown. Thus no specific treatment is available. An animal model of this disease would allow for testing of new treatments. Because of the observed elevations in serum short-chain fatty acid concentrations $(8,13)$ in these patients and the extensive fat accumulation in the liver, infusion of the short-chain fatty acid sodium octanoate into experimental animals was used to try to develop an experimental model.

\section{MATERIALS AND METHODS}

Male 2-kg albino rabbits were anesthetized with sodium pentobarbital $5 \mathrm{mg} / \mathrm{kg}$. Catheters were placed in the carotid artery and ear vein. Heart rate, respiratory rate, blood pressure, and electroencephalogram were monitored continuously during the experiment using a Grass 4-channel polygraph. When the animals awoke and vital signs stabilized, intravenous infusion of $0.2 \mathrm{M}$ sodium octanoate $\mathrm{pH} 7.4$ was begun. Blood was drawn hourly for biochemical studies as described previously (14). After $4 \mathrm{~h}$ of continuous infusion of octanoate, the animals were given a rapid intravenous injection of $100 \mathrm{mg}$ pentobarbital. The heart was exposed and the rabbits perfused first with normal saline and then with Karnovsky solution. Liver and brain were removed and prepared for histologic and ultrastructural examination. Heart, kidney, and lungs were also removed and examined histologically. In some animals, sections of these organs were removed before perfusion for frozen sections and oil red $\mathrm{O}$ stains. Brains of some rabbits were removed before perfusion for determination of octopamine concentrations using the method of Saavedra (11).

\section{RESULTS}

Physiologic changes. During octanoate infusion, rabbits developed hypotonia, coma, and pupillary dilitation. Seizures were observed in approximately $1 / 3$ of the rabbits (Fig. 1). Significant hyperventilation with respiratory alkalosis developed during octanoate infusion (Table 1)

Biochemical changes (14). Blood ammonia concentrations rose $300 \%$, from a control mean of $39 \pm 5 \mu \mathrm{g} \%$ to a $4 \mathrm{~h}$ maximum of $119 \pm 44(P<0.025)$. Serum glutamic oxaloacetic transaminase (SGOT) rose $143 \%$ from 46 to 66 but this was not statistically significant. Serum lactic acid concentrations rose 183\% (1.73-3.16 $\mu \mathrm{M} /$ liter, $P<0.025$ ). Brain octopamine concentrations rose from a control mean of $4.22 \mathrm{pg} / \mu \mathrm{g}$ protein to a $4 \mathrm{~h}$ mean of $12.61(P$ $<0.005)$.

Pathologic changes in liver were found after $4 \mathrm{~h}$ of infusion. The liver architecture was intact and there was no evidence of hepatocellular necrosis or inflammatory reaction. Microvesicular fatty accumulation was observed throughout the liver, and primarily around the portal vein (Fig. $2 \mathrm{~A}$ ). Vacuolization of the cytoplasm of hepatocytes was also observed. Particularly in cells surrounding the portal vein, hepatocytes appeared pale and swollen. Ultrastructural alterations in the liver were confined primarily to mitochondria, which were swollen, pale and more pleiomorphic in appearance than mitochondria of control animals (Fig. 2B). There was a "fluffy" appearance to the matrix. The cytoplasm contained an increased number of lipid droplets. There was an increased incidence of autophagic vacuoles in the cytoplasm. No other ultrastructural changes were noted.

In the brain there was no gross evidence of cerebral edema. The only histologic abnormality noted was the appearance of early chromatolysis in scattered neurons of the brain stem, with swollen cells, peripherally placed nuclei, and displacement of Nissl substance towards the periphery (Fig. 3). Although no consistent changes were noted on ultrastructural examination, occasionally swelling of the astrocytic foot processes was observed, suggesting early cerebral edema.

Examination of the heart, kidney and lungs revealed mild lipid accumulation in these organs and no other structural abnormalities.

\section{DISCUSSION}

Continuous infusion of octanoate into animals produces clinical, biochemical, and pathologic alterations which are strikingly similar to those encountered in patients with Reye's syndrome. In both cases, seizures, coma, and hyperventilation are prominent, as are hyperammonemia, lactic acidemia, and respiratory alkalosis $(6,14)$. Elevated concentrations of octopamine, a "false" neurotransmitter, have been found in brains of patients with Reye's syndrome (7). Similar elevations were present in rabbits after octanoate infusion. 


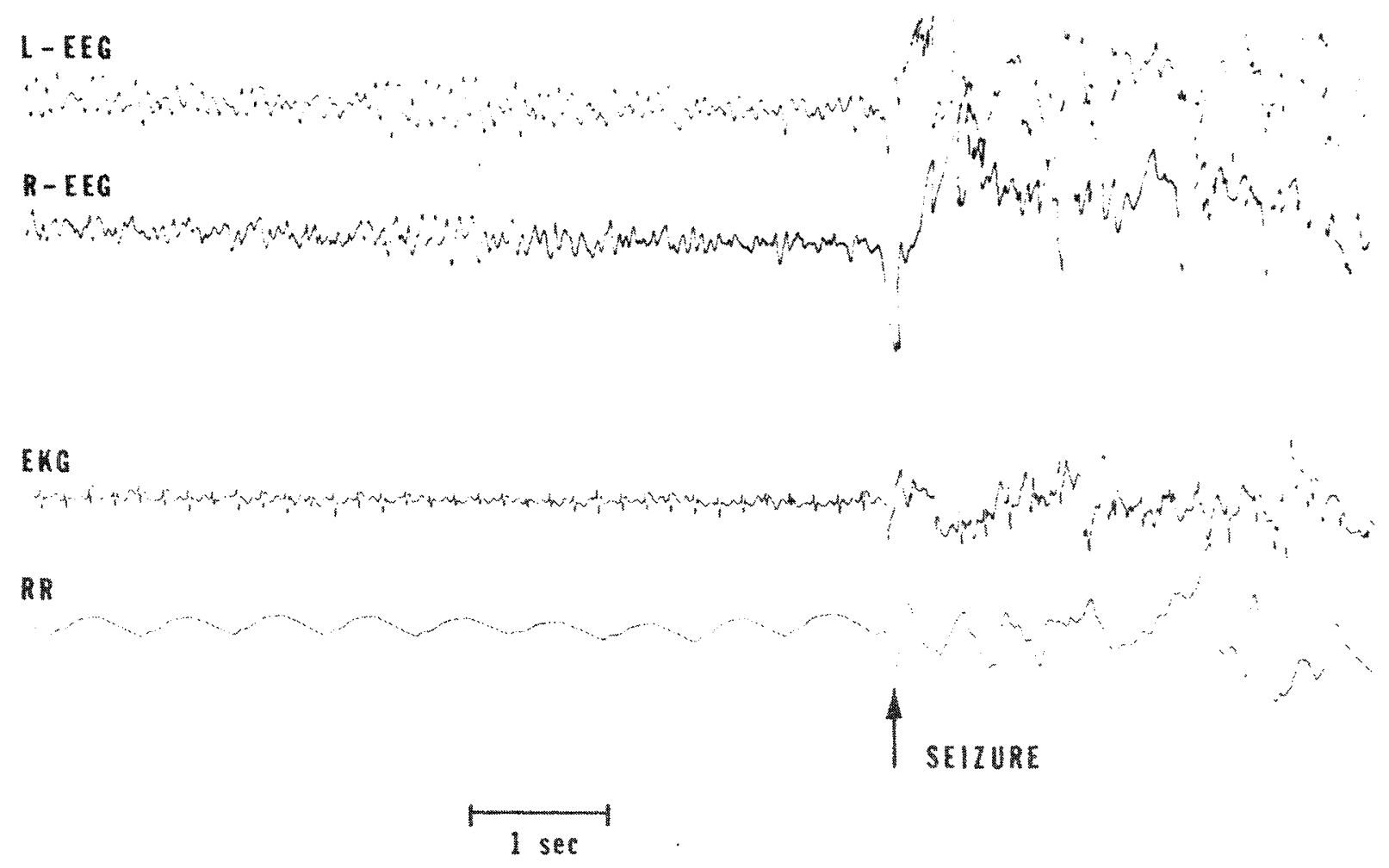

Fig. 1. Polygraph recording of EEG, EKG, and respiratory rate (RR) in rabbit during infusion of octanoate. At $1 \mathrm{~h}$ of infusion, the rabbit developed clinical and electrographic seizure activity, which lasted approximately $30 \mathrm{sec}$.

Table 1. Physiologic data during octanoate infusion

\begin{tabular}{lrcccc}
\hline \multicolumn{1}{c}{ Time $(\mathrm{h})$} & 0 & 1 & 2 & 3 & 4 \\
\hline Mean respiratory rate & 92 & 131 & 150 & 199 & 237 \\
Mean heart rate & 275 & 289 & 305 & 295 & 296 \\
Mean blood pressure & 67 & 70 & 67 & 66 & 62 \\
Mean pH & 7.48 & 7.53 & 7.53 & 7.48 & 7.48 \\
Mean $\mathrm{Po}_{2}$ & 95 & 98 & 84 & 80 & 85 \\
Mean $\mathrm{PCO}_{2}$ & 26 & 20 & 16 & 19 & 19 \\
\hline
\end{tabular}

Pathologic changes in Reye's syndrome consist primarily of microvesicular fatty accumulation in the liver, mitochondrial swelling (9), and nonspecific changes of cerebral edema in the brain. The experimental model reproduces the fatty accumulation in liver and mitochondrial swelling. Although obvious cerebral edema was not observed, ultrastructural evidence of swelling of astrocytic foot processes suggests that cerebral edema may have been developing. Moreover, recent studies from this laboratory have demonstrated that a significant elevation in intracranial pressure occurs during octanoate infusion (15).

Several other animal models for Reye's syndrome have been proposed $(2-5)$. The one most closely related to the octanoate model is produced by injection of 4-pentenoic acid into rats (5). 4-Pentenoic acid is a short-chain fatty acid analogue of hypoglycin A (1), the presumed exogenous toxin which causes Jamaican vomiting sickness, a disorder clinically similar to Reye's syndrome. Intraperitoneal injection of 4-pentenoic acid (5) produces fatty accumulation in liver and swelling of hepatocytes, elevations in plasma ammonia and serum glutamic-oxaloacetic transaminase concentrations, hypoglycemia in fasted rats and hyperglycemia in fed rats. Free fatty acid levels do not change after a single injection but decrease after repeated injections of pentenoic acid.

Although this model reproduces some of the abnormalities found in Reye's syndrome, there are several problems which make it less desirable than the octanoate model. 4-Pentenoic acid is not a naturally occurring fatty acid in humans, whereas octanoate is: furthermore, serum octanoate concentrations are elevated in patients with Reye's syndrome (8). Thus far there has been no evidence of 4-pentenoic acid accumulation in the human disorder. Finally, in contrast to patients with Reye's syndrome, total free fatty acid levels in pentenoic acid-treated rats do not rise significantly, and in fact decrease during chronic studies. The authors suggest that it is unlikely that exposure to pentenoic acid or related compounds causes Reye's syndrome, but rather the two conditions share similar biochemical defects. One effect of pentenoic acid is to block fatty acid oxidation (5). Although specific short-chain fatty acid concentrations have not been measured after pentenoic acid injection, it is possible that this block in fatty acid oxidation leads to accumulation of fatty acids such as octanoate, which in turn produce the clinicopathologic syndrome observed in the rats. In Reye's syndrome the cause of the organic acidemia is unclear, but one possible mechanism is a block in beta oxidation of fatty acids e.g., by a virus either alone or in combination with an exogenous toxin. The resultant organic acid accumulation could then produce the clinical, biochemical, and pathologic abnormalities observed in the human disease. Of significance is the fact that serum concentrations of octanoate capable of reproducing this syndrome in rabbits are of the same order of magnitude as those found in children with acute Reye's syndrome (14). One criticism of the "fatty acid" hypothesis is that concentrations of short-chain fatty acids are much lower than those found in patients with inborn errors of organic acid metabolism such as isovaleric acidemia (12). However, it is possible that rapid accumulation of short-chain fatty acids in previously healthy children causes more toxicity than chronic accumulation of these substances, whereas children with long-term organic acidemia may be able to adapt metabolically to these changes.

It is not clear whether the short-chain fatty acidemia in Reye's syndrome contributes to the encephalopathy and other abnormalities found in the disease. At least in this experimental model, infusion of the short-chain fatty acid octanoate is capable of 

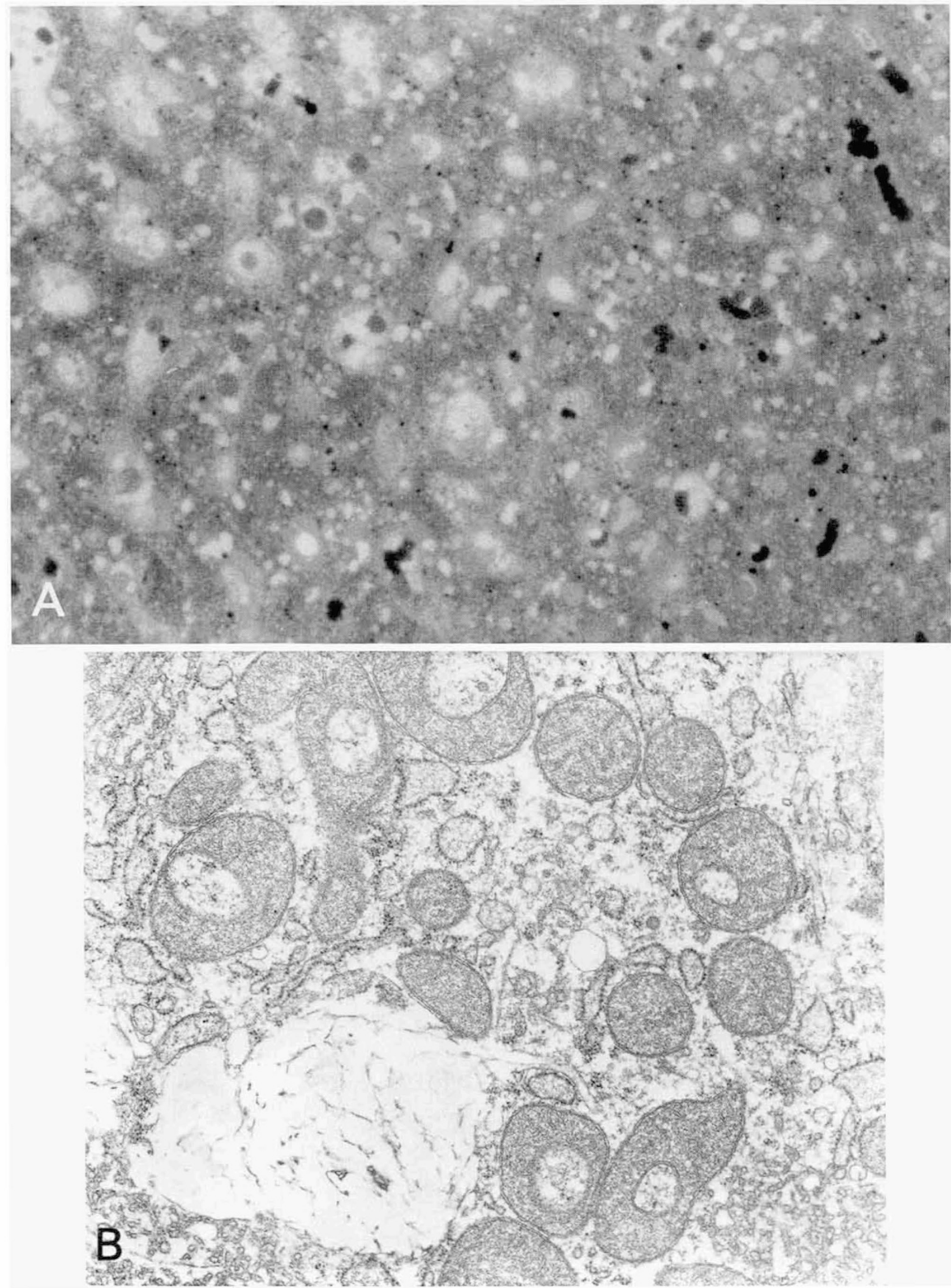

Fig. 2A. Thick section of rabbit liver following 4-h infusion of $0.2 \mathrm{M}$ sodium octanoate; stained with toluidine blue. Note diffuse fatty infiltration of hepatocytes, $\times 450$.

Fig. 2B. Electron micrograph of liver from rabbit given 4-h infusion of octanoate. Note pleimorphic changes in mitochondria and "fluffy" appearance of mitochondrial matrix, $\times 15,000$. 


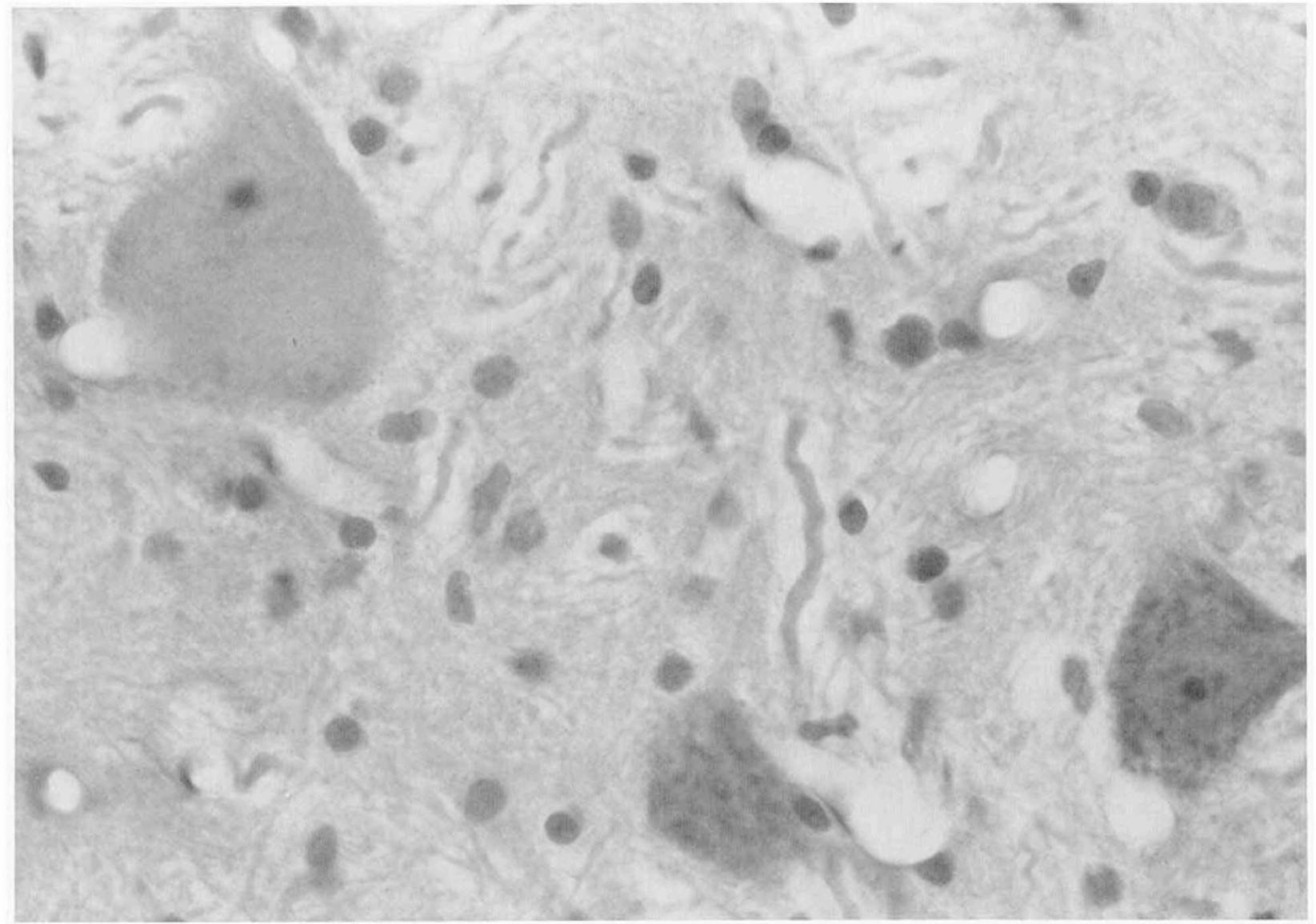

Fig. 3. Section of rabbit brainstem after 4-h octanoate infusion. Note swollen pale neuron with peripheral displacement of nucleus. H and E stain, $\times 500$

reproducing the major clinical, biochemical, and pathologic alterations found in patients with Reye's syndrome. It may serve as a useful model with which to study the effects of various therapeutic agents in preventing or reversing the disease process.

\section{REFERENCES AND NOTES}

1. Bressler, R., Corredor, C., and Brendel, K.: Hypoglycin and hypoglycin-like compounds. Pharmacol. Rev., 21: 150 (1969)

2. Colon, A. R., Ledesma, F., Pardo, V., and Sandberg, D. H.: Viral potentiation of chemical toxins in the experimental syndrome of hypoglycemia, encephalopathy, and visceral fatty degeneration. Digestive Dis., 19: 1091 (1974).

3. Crocker, J. F. S., Ozere, R. L., Rozee, K. R., Digout, S. C., and Hutzinger, O.: Insecticide and viral interaction as a cause of fatty visceral changes and encephalopathy in the mouse. Lancet, 2: 22 (1974).

4. Davis, L. E., Cole, L. L., and Kornfeld, M.: Experimental model of influenza virus toxicity: a potential model for Reye's syndrome. Neurology, 4(Suppl.): 142 (1981).

5. Glasgow. A. M. and Chase, H. P.: Production of the features of Reye's syndrome in rats with 4-pentenoic acid. Pediatr. Res., 9: 133 (1975).

6. Huttenlocher, P. R. and Trauner, D. A.: Reye's Syndrome. In: P. K. Vinken and G. W. Bruyn, Eds.: Handbook of Clinical Neurology. V. 29, pp. 331-44. (No. Holland Publ. Co., Amsterdam, 1977).

7. Lloyd, K. G., Davidson, L., Price, K., McClung, H. J.. and Gall. D. G.: Catecholamine and octopamine concentrations in brains of patients with Reye syndrome. Neurology, 27: 985 (1977).

8. Mamunes, P., DeVries, C. H., Miller, C. O.. and David, R. B.: Fatty acid quantitations in Reye's syndrome. In: J. D. Pollack, Ed.: Reye's Syndrome. pp. 245-54. (New York, Grune and Stratton, 1975).

9. Partin, J. C., Schubert, W. K., and Partin. J. S.: Mitochondrial Ultrastructure in Reye's Syndrome. N. Engl. J. Med., 285: 1339 (1971)

10. Reye, R. D. K., Morgan, G., and Baral, J.: Encephalopathy and fatty degeneration of the viscera. A disease entity in childhood. Lancet, 2: 749 (1963).

11. Saavedra, J. M.: Enzymatic-isotopic method for octopamine at the picogram level. Anal. Biochem., 59:628 (1974).

12. Tanaka, K.: Jamaican vomiting sickness and Reye's syndrome. N. Engl. J. Med. 295: 1481 (1976).

13. Trauner, D. A., Nyhan, W. L., and Sweetman, L.: Short-chain organic acidemia and Reye's syndrome. Neurology, 25: 296 (1975).

14. Trauner, D. A. and Huttenlocher, P. R.: Short-chain fatty acid-induced central hyperventilation in rabbits. Neurology, 28: 94-5 (1978).

15. Trauner, D. A. and Adams, H.: Intracranial pressure elevation during octanoate infusion in rabbits. Pediatr. Res., 15: 1097 (1981).

16. Varma, R. R., Riedel, D. R., Komorowski. R. A., Harrington, G. J., and Nowak, T. V.: Reye's syndrome in nonpediatric age groups. J. Am. Med. Assoc., 242: 1373 (1979).

17. The author thanks Drs. Henry C. Powell and Katsumi Miyai for reviewing photomicrographs, Mr. Michael Costello and Ms. Heather Adams for technical assistance and Ms. Valerie Brainard for manuscript preparation.

18. Requests for reprints should be addressed to: Dr. Doris A. Trauner. Department of Neurology H-815, University Hospital, San Diego, California 92103.

19. This research was funded in part by a grant from the National Reye's Syndrome Foundation of Bryan, Ohio and by Basic Research Grant \#1-699 from March of Dimes Birth Defects Foundation.

20. Received for publication August 13,1981.

21. Accepted for publication April 21, 1982. 